\title{
ELECTROMAGNETIC INVERSION FOR ENVIRONMENTAL SITE CHARACTERIZATION : DATA QUALITY VERSUS IMAGE RESOLUTION
}

\author{
David L. Alumbaugh and Gregory A. Newman \\ Geophysics Department, PO Box 5800,MS 0750 \\ Sandia National Laboratories, Albuquerque, NM 87185
}

\begin{abstract}
Non-linear electromagnetic inversion schemes have been developed to produce 2D and 3D images of subsurface conductivity structure from electromagnetic geophysical data. The solutions are obtained by successive linearized model updates where full forward modeling is employed at each iteration to compute model sensitivities and predicted data. Regularization is applied to the problem to provide stability. The use of the inversion is demonstrated on a data set collected with the Apex Parametrics 'MaxMin I-8S' over a section of conductive waste at the Idaho National Laboratory's 'Cold Test Pit'. The out-of phase data are of very good quality while the in-phase are rather noisy due to slight mispositioning errors. A resolution study on synthetic data indicates that the error present in the in-phase data causes images of far lower resolution with more artifacts than if the in-phase and out-of-phase components are of similar quality. Better resolution images result if the data are weighted proportional to frequency; this gives each frequency equal weight. The loss of resolution due to poor quality in-phase data is demonstrated in a 3D inversion of the MaxMin data which shows both artifacts forming outside of the area known to contain the buried waste, as well as an inability to resolve depths.
\end{abstract}

\section{INTRODUCTION}

Frequency domain, controlled source electromagnetic methods are routinely employed to map the location of barrels, trenches and pits, metals, plumes, etc., at hazardous waste sites. Although the raw data may provide excellent resolution regarding the horizontal location of these targets, it directly yields very little information about their vertical extent and often cannot define sharp boundaries. In order to produce high resolution images of the subsurface conductivity structure, non-linear inversion or approximate imaging techniques must be applied to the data. Here we employ a full non-linear inversion scheme implemented for both $2.5 \mathrm{D}$ and 3D geometries (Newman and Alumbaugh, 1997). After an introduction to the schemes we give a brief description of the Apex Parametrics MaxMin I- 8S data collection system and follow this with a 2.5D synthetic example of how different data noise levels effect the images. Finally we show results from a 3D inversion of data collected over the Cold Test Pit at the Idaho National Engineering Laboratory in June of 1996.

\section{THE INVERSE SOLUTION}

The parameterization used in the inverse solutions will be kept sufficiently fine because we are interested in reconstructions that do not under parameterize the earth. This forces the inverse problem to be underdetermined, which makes it unstable and ill posed. Reliable estimates of the model parameters $\mathbf{m}$ may be possible if a least squares inversion is stabilized with regularization (Tikhonov and Arsenin, 1977) which provides for reconstructions that are smoothly varying from one point to another. This smoothly varying nature occurs at the expense of an increase in the fitting error.

Forming a least squares functional, linearizing it about a given earth model, $\mathbf{m}^{(\mathbf{i})}$, at a given iteration $i$, and minimizing the result yields an equation for the model update:

$$
\mathbf{m}=\left[\left(\mathbf{D A}^{\mathbf{p}(\mathbf{i})}\right)^{\mathbf{T}}\left(\mathbf{D} \mathbf{A}^{\mathbf{p ( i )}}\right)+\lambda \mathbf{W}^{\mathbf{T}} \mathbf{W}\right]^{-1}\left(\mathbf{D} \mathbf{A}^{\mathbf{p}(\mathbf{i})}\right)^{\mathbf{T}} \mathbf{D} \delta \mathbf{d}^{(\mathbf{i})}
$$


where

$$
\delta \mathbf{d}^{(\mathbf{i})}=\mathbf{d}-\mathbf{d}^{\mathbf{p}(\mathbf{i})}+\mathbf{A}^{\mathbf{p}(\mathbf{i})} \mathbf{m}^{(\mathbf{i})} .
$$

The idea is through the proper choice of the tradeoff parameter,$\lambda$, find the smoothest model that fits the data within the estimated data error. The roughness or regularization matrix (W) consists of a finite difference approximation to the Laplacian operator, the observed data are represented by the vector $\mathbf{d}$, and the predicted data arising from the model $\mathbf{m}^{(\mathbf{i})}$ at the $i^{\prime}$ th iteration of the inversion procedure are denoted by $\mathbf{d} \mathbf{p}(\mathbf{i})$. These values are calculated using the finite differences scheme given in Alumbaugh et al. (1996). The data weighting matrix $\mathbf{D}$ is diagonal and consists of individual estimates of the data noise. The Jacobian or model sensitivities matrix is also computed using the finite difference scheme and is given by $\mathbf{A}^{\mathbf{p}(\mathbf{i})}$.

The iterative procedure is initiated by assuming some initial background model, where we compute the predicted data and electric field for all transmitter and unique receiver locations. At the first iteration we calculate the model update via equation (1) with the appropriate selection of the tradeoff parameter to provide a smooth model (see Newman and Alumbaugh (1997) for more details on how this parameter is chosen). After the model update is determined, the predicted data are computed and the residual errors between the predicted and measured data calculated. If the sum of the squared residual errors is less that of the estimated data noise, or if the number of iterations has obtained some predefined limit, the process is terminated. Otherwise the scheme proceeds to the next iteration where it linearizes about the new model $\mathbf{m}$, computes the electric fields for the updated background model and determines the new model update with a reduced value of the tradeoff parameter which allows for better image resolution.

\section{DESCRIPTION OF THE GEOPHYSICAL SURVEY AND DATA}

The data analyzed in this study were collected with the Apex Parametrics MaxMin I-8S frequency domain system (Apex Parametrics, 1994) at the Idaho National Engineering Laboratory's Cold Test Pit (CTP) as part of the DOE sponsored Electromagnetic Integrated Demonstration (EMID) project (Pellerin et al, 1997). The MaxMin I-8S system is a loop-loop frequency domain system consisting of a small transmitter loop (approximately $0.5 \mathrm{~m}$ on a side) connected to a ferrite coil receiver system by a reference cable of various length. Coil separations of $4.57,9.14$ and 18.28 meters were employed during the survey using eight frequencies ranging from $440 \mathrm{~Hz}$ to $56320 \mathrm{~Hz}$ in bilinear steps; here we will only examine the $9.14 \mathrm{~m}$ separation. The in-phase and out-of-phase components of the scattered magnetic field are measured in terms of percentage of the free space or primary field.

The in-phase primary or free-space field produced by the transmitter is canceled at the receiver by "bleeding" current off of the transmitter to exactly oppose the current induced by this unwanted component in the receiver. This causes the measured in-phase scattered field to be very sensitive to errors in the source-receiver separation. For example, a change in positioning of $3 \mathrm{~cm}$ between coils separated by $9.14 \mathrm{~m}$ produces an error of approximately $1 \%$ of the primary field. However, this error in separation causes negligible changes in the out-ofphase component. Thus the in-phase data are of much poorer quality than the out-of-phase, and since we weight the data by estimates of the noise ( $D$ in equation 1 ), the in-phase data are going to have much less effect on the resulting images compared to the out-of-phase data.

The survey was limited to the four primary lines of the CTP (See Figure 1 in Pellerin et al., 1997). For the $9.14 \mathrm{~m}$ coil separation a $1.52 \mathrm{~m}$ sampling was employed for every line. The receiver unit was maintained at a height of approximately $1.3 \mathrm{~m}$ off the ground, while transmitter coil was $0.79 \mathrm{~m}$ off the ground.

\section{RESOLUTION LIMITS DUE TO NOISY IN-PHASE DATA}

To demonstrate the problems caused by noisy in-phase data, a simulation of the CTP survey was calculated for two $0.1 \mathrm{~S} / \mathrm{m}$ blocks $(9 \mathrm{~m}$ wide by $5 \mathrm{~m}$ tall) separated by $9 \mathrm{~m}$ and buried at $3 \mathrm{~m}$ depth in a $0.01 \mathrm{~S} / \mathrm{m}$ half-space using the scheme described in Alumbaugh et al. (1996). A data sampling interval of $3 \mathrm{~m}$ was used with a source-receiver separation of $10 \mathrm{~m}$, and following the specifications of the MaxMin system eight frequencies were employed. In the first case the data noise level was assumed to be $0.01 \%$ of the primary field in both the real and quadrature 
components (Figure 1a), while in the second case it was assumed that the noise in the in-phase component was $2 \%$ of the primary, which is a more realistic assumption (Figure $1 \mathrm{~b}$ ).

As can be seen in Figure 1a, with a $0.01 \%$ noise estimate we are able to recover the general location of the two bodies, and get a crude representation of the fact that there is material overlying the bodies. However notice that the bottom of the two bodies are smeared somewhat. Using more realistic estimates of the noise in the in-phase component (Figure 1b) severely degrades the resolution of the image. Although we can tell that there are two bodies present, the locations and size are severely distorted. Also we are not able to resolve the capping layer over the bodies. Finally notice that a large amount of asymmetry has been introduced. Although we do not have a definite reason for this phenomenon, we believe it is due to the fact that the survey configuration was not symmetric over the bodies.

A third way to weight the data is to make the weighing parameters proportional to the frequency. Although it may be unrealistic since higher frequencies yield more scattering which improves the signal to noise ratio, this method gives equal weight to each frequency and thus the lower frequency data are not dominated by the higher frequencies. In Figure 1c results are plotted for this type of weighting such that the lowest frequency $(440 \mathrm{~Hz})$ has a data error of $8.3 \times 10^{-4} \%$ of the primary field in both the in-phase and out-of-phase components, while for the highest frequency $(56320 \mathrm{~Hz})$ the error is $0.11 \%$. It can be seen that better depth resolution results, especially in the left body.
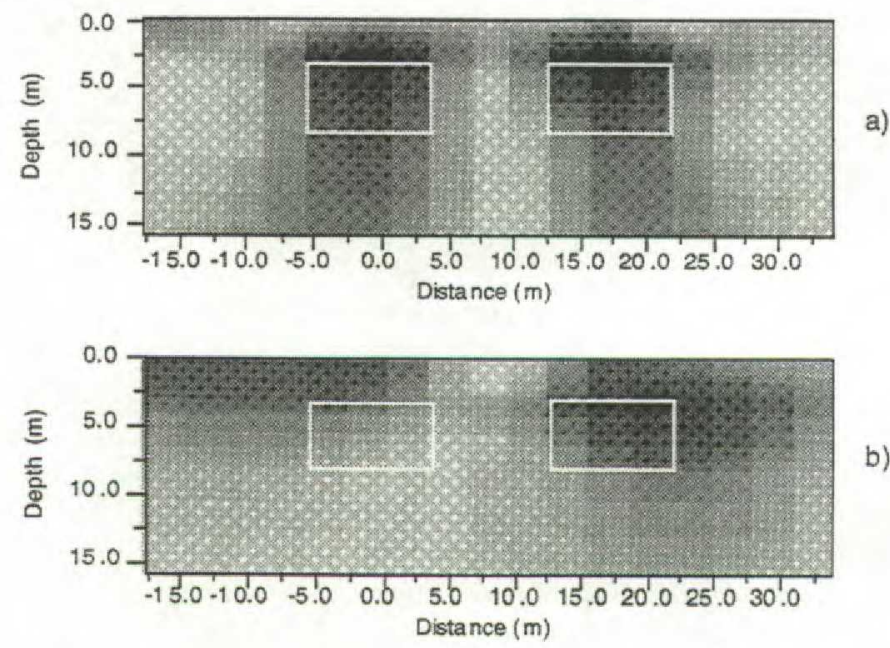

a)

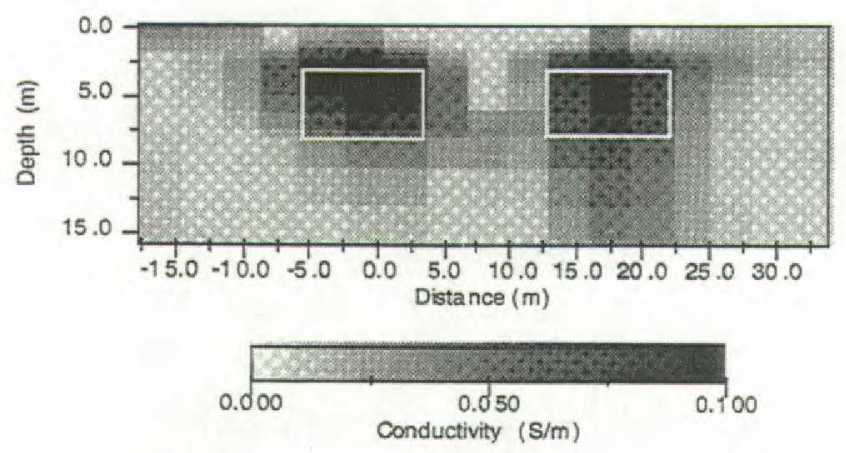

Figure $1-2.5 \mathrm{D}$ resolution analysis for different assumed error levels. The white boxes locate the two $0.1 \mathrm{~S} / \mathrm{m}$ boxes located in a $0.01 \mathrm{~S} / \mathrm{m}$ background. a) In-phase error $=0.01 \%$ of primary field. b) In-phase error $=2.0 \%$ of primary field. c) In-phase and out-of-phase errors are proportional to the frequency.

\section{3-D INVERSION RESULTS}

Figure 2 shows an image slice through the center of the CTP survey area for the $9.14 \mathrm{~m}$ offset data. Notice that the system is detecting the basalt beneath the pit through the fill material. 
However, also notice that 1 ) we can not detect the more resistive capping material covering the pit as the near surface is very conductive, and 2) an anomaly at depth extends outside of the pit area. As demonstrated above, this most likely is due to the fact that the noise in the real component was estimated to be $2 \%$ while the out-of-phase was much less $(0.1 \%)$.

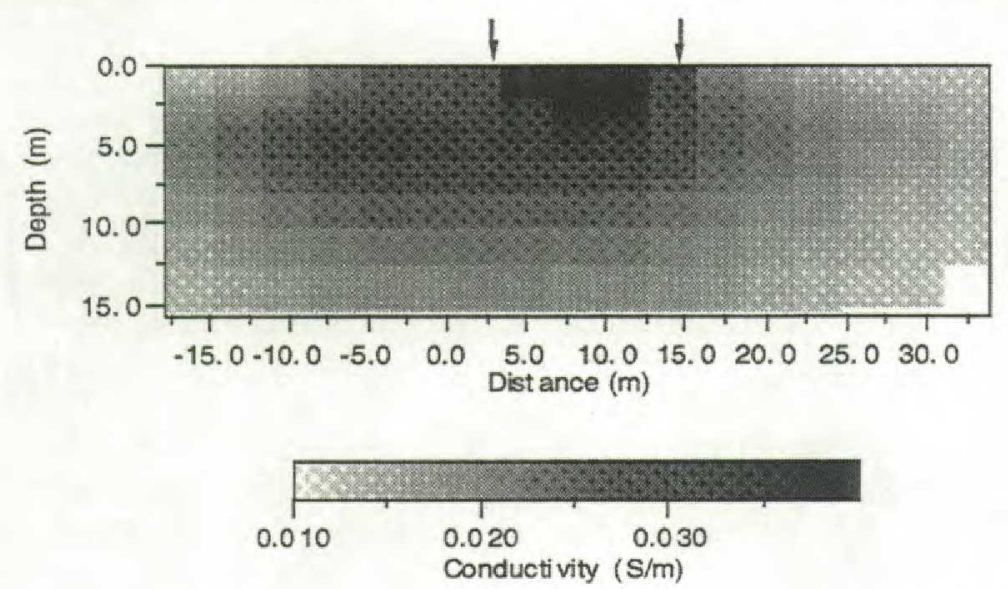

Figure 2 - 3D inversion results through the center of the survey area for the $9.14 \mathrm{~m}$ offset MaxMin data. The arrows designate the assumed location of the pit edges.

\section{CONCLUSIONS}

The results above show problems that are associated with inversion of frequency domain data collected with standard loop-loop systems. Although better quality data could be collected with systems that use coils mounted on a fixed boom, experience has shown that for examples like the one presented here the small coil separation will not provide adequate penetration to detect the resistive material below the conductive fill. One method of getting around the problem of the primary field is collecting very accurate time domain data that can either be accurately transformed to the frequency domain or inverted directly in the time domain. Also we are currently completing a scheme to invert impedance data so that AMT and RMT data can be analyzed. This removes the source problems encountered in this study.

\section{ACKNOWLEDGMENTS}

This work was performed at Sandia National Laboratories, which is operated for the U.S. Department of Energy. Funding for this project was provided by DOE's office of Basic Energy Sciences, Division of Engineering and Geoscience under contract DE-AC0494AL85000. The data were collected David Alumbaugh and Louise Pellerin under the VETEM project which was supported by the DOE Office of Technology Development.

\section{REFERENCES}

Alumbaugh, D.L., Newman, G.A., Prevost, L., and Shadid, J.N., 1996, Three-dimensional wide band electromagnetic modeling on massively parallel computers: Radio Science, 31, 1-23.

Apex Parametrics Ltd.,1994, Apex MaxMin I-10 and I-8S EM Systems Operations Manual: Apex Parametrics, Uxbridge, Ontario, Canada.

Newman, G.A., and Alumbaugh, D.L., 1997, 3-D massively parallel electromagnetic inversion; part a - theory: Geophy. J. Int.,128,345-354.

Pellerin, L, Alumbaugh, D.L, and Pfeifer, M.C., 1997, The electromagnetic integrated demonstration at the Idaho National Engineering Laboratory Cold Test Pit: Proceedings of SAGEEP '97 (Symposium on the Application of Geophysics to Environmental and Engineering Problems ), Reno, Nevada.

Tikhonov, A. N. and Arsenin, V. Y., 1977, Solutions to ill-posed problems: John Wiley and Sons, Inc. 